\title{
Genome-wide comparative analysis of type-A Arabidopsis response regulator genes by overexpression studies re- veals their diverse roles and regulatory mechanisms in cytokinin signaling
}

\author{
Bo Ren ${ }^{1,2}$, Yan Liang ${ }^{1}$, Yan Deng ${ }^{1,2}$, Qingguo Chen ${ }^{1,2}$, Jian Zhang ${ }^{1}$, Xiaohui Yang $^{1}$, Jianru Zuo \\ ${ }^{1}$ State Key Laboratory of Plant Genomics and National Plant Gene Research Center (Beijing), Institute of Genetics and Develop- \\ mental Biology, Chinese Academy of Sciences, Beijing 100101, China; ${ }^{2}$ Graduate School, Chinese Academy of Sciences, Beijing \\ 100049, China
}

Cytokinin is a critical growth regulator for various aspects of plant growth and development. In Arabidopsis, cytokinin signaling is mediated by a two-component system-based phosphorelay that transmits a signal from the receptors, through histidine phosphotransfer proteins, to the downstream response regulators (ARRs). Of these $A R R s$, type-A $A R R$ genes, whose transcription can be rapidly induced by cytokinin, act as negative regulators of cytokinin signaling. However, because of functional redundancy, the function of type-A $A R R$ genes in plant growth and development is not well understood by analyzing loss-of-function mutants. In this study, we performed a comparative functional study on all ten type-A $A R R$ genes by analyzing transgenic plants overexpressing these $A R R$ genes fused to a MYC epitope tag. Overexpression of $A R R$ genes results in a variety of cytokinin-associated phenotypes. Notably, overexpression of different $A R R$ transgenes causes diverse phenotypes, even between phylogenetically closely-related gene pairs, such as within the $A R R 3-A R R 4$ and $A R R 5-A R R 6$ pairs. We found that the accumulation of a subset of ARR proteins (ARR3, ARR5, ARR7, ARR16 and ARR17; possibly ARR8 and ARR15) is increased by MG132, a specific proteasomal inhibitor, indicating that stability of these proteins is regulated by proteasomal degradation. Moreover, similar to that of previously characterized ARR5, ARR6 and ARR7, stability of ARR16 and ARR17, possibly including ARR8 and ARR15, is regulated by cytokinin. These results suggest that type-A ARR proteins are regulated by a combinatorial mechanism involving both the cytokinin and proteasome pathways, thereby executing distinctive functions in plant growth and development.

Keywords: Arabidopsis, cytokinin, MG132, protein stability, type-A ARR

Cell Research (2009) 19:1178-1190. doi: 10.1038/cr.2009.88; published online 21 July 2009

\section{Introduction}

Cytokinin is a classical phytohormone that plays important roles in many aspects of plant growth and development. Cytokinin regulates embryo and seed development, stem-cell specification, shoot and root development, flowering time, leaf senescence and the circadian clock. Generally, it is believed that cytokinin acts to

Correspondence: Jianru Zuo

Tel: +86-10-6486 3356; Fax: +86-10-6487 3428

E-mail: jrzuo@genetics.ac.cn

Received 9 June 2009; revised 11 June 2009; accepted 12 June 2009; published online 21 July 2009 stimulate cell division and cell differentiation $[1,2]$. In Arabidopsis thaliana, genetic and biochemical studies have defined major components of the cytokinin signaling pathway. According to our current understanding, cytokinin signaling is transduced through a phosphorelay based on a two-component system (TCS). In Arabidopsis, the TCS mainly consists of Histidine Kinases (AHKs), Histidine Phosphotransfer Proteins (AHPs) and Response Regulators (ARRs) [3-8]. Three AHKs, AHK2, 3 and 4, have been characterized as cytokinin receptors, of which AHK4 has also been known as CYTOKININ RESPONSE 1 (CRE1) or WOODEN LEG (WOL) [914]. In the presence of cytokinin, the receptors are autophosphorylated at a conserved His residue and then 
transfer the phosphoryl group to a conserved Asp residue $[12,15]$. This phosphoryl group is subsequently transferred to a conserved His residue of AHP proteins, which consist of five members named AHP1 through AHP5. Upon phosphorylation, AHPs are rapidly shuttled from the cytoplasm into the nucleus, where ARR proteins are activated upon receiving a phosphoryl group from AHPs [16-19]. When type-B ARRs, a class of MYB-type transcription factors, receive the phosphoryl group from AHPs, they activate expression of downstream type-A $A R R$ genes. Type-A ARRs, in turn, act to repress the activity of type-B ARRs by a negative feedback mechanism $[4,5,18,20]$.

In the Arabidopsis genome, 24 ARR genes have been identified, which were mainly classified into two groups based on their expression patterns, structures and possible functions of the encoded proteins [21-25]. Type-B ARRs, including ARR1, 2, 10 14, 18 21 and 23, contain a receiver domain and a long C-terminal extension carrying a DNA-binding domain named GARP [23, 24, 26]. Mutations in single type-B $A R R$ genes do not show apparent phenotype, whereas multiple mutants show reduced sensitivities to cytokinin and a variety of developmental defects, suggesting that this group of transcription factor genes functions redundantly [27-30]. Moreover, overexpression of a dominant transcriptional repressor $A R R 1-S R D K$ results in the suppression of most, if not all, activities of type-B $A R R$ genes, which consequently causes a cytokinin-deficiency phenotype [31]. Similarly, induced ubiquitous expression of the dominant transcriptional repressor ARR10-EAR in early embryogenesis causes defective pattern formation [32]. Conversely, overexpression of several type-B $A R R$ genes results in typical cytokinin responses $[20,33,34]$. These observations indicate that type-B $A R R$ genes function as positive regulators of cytokinin signaling.

Type-A ARR proteins, including 10 typical members (ARR3 9, 15 17) and 2 atypical members (ARR22 and 24), contain a receiver domain at the $\mathrm{N}$-terminal region and a short variable extension at the $\mathrm{C}$-terminal region $[23,24]$. Typical type-A $A R R$ genes are primary responsive genes in cytokinin signaling, and their expression can be rapidly induced by cytokinin in the absence of $d e$ novo protein synthesis $[35,36]$. A major function of typeA ARR proteins is to act as negative regulators of cytokinin signaling by repressing type-B ARRs via unknown mechanisms. In addition, type-A $A R R$ genes have also been shown to play multiple roles in plant growth and development. For example, ARR3, ARR4 and ARR 9 are involved in the regulation of the circadian rhythm [3739]. ARR4 acts as a molecular node integrating light and cytokinin signaling by interacting with red light receptor phytochrome B [40]. ARR5, ARR6, ARR7 and ARR15 are repressed by WUSCHEL to control meristem development [41]. ARR7 and ARR15 are also shown to be positively regulated by auxin and play a role in root stem-cell niche specification [32]. However, little is known about the biochemical mechanism of the type-A ARR protein activity.

A challenge in genetic studies on ARR has been encountered by functional redundancy among those genes. Most, if not all, mutations in single type-A ARR genes do not cause obvious abnormalities and altered responses to cytokinin. In a remarkable effort, Kieber and coworkers [42] have generated multiple mutants of type-A ARR genes, most of which, including a hextuple mutant (arr3, $4,5,6,8,9)$, exhibited only minor differences compared with wild-type plants under normal growth conditions. As an alternative approach, analysis of the gain-of-function phenotype has yielded valuable information on the function of type-A $A R R$ genes. Overexpression of several type-A $A R R$ genes causes reduced sensitivity to cytokinin in root elongation and shoot formation assays or an early flowering phenotype [43-46]. Interestingly, several type-A ARR proteins have been found to be stabilized by cytokinin in a phosphorelay-dependent manner, thus uncovering an additional regulatory mechanism of cytokinin signaling [46]. In this study, we performed a genomewide analysis of all type-A $A R R$ genes by the generation and characterization of transgenic Arabidopsis plants overexpressing individual type-A $A R R$ genes. We found that the type-A $A R R$ genes show distinctive functions in plant growth and development, and that stability of some type-A ARR proteins is likely regulated by cytokinin and proteasome machinery. These results provide new insight into the regulatory mechanism of cytokinin signaling.

\section{Results}

Generation of transgenic Arabidopsis plants overexpressing type- $A$ ARR genes

To facilitate functional analysis, we have generated transgenic plants overexpressing type-A $A R R$ genes (ARR3, 4, 5, 6, 7, 8, 9, 15, 16 and 17). cDNA fragments containing respective coding sequences of individual $A R R$ genes were obtained by reverse transcription (RT)PCR, in which the stop codons were removed during $\mathrm{PCR}$. The cDNA fragments were fused in-frame to a $6 \mathrm{X}$ MYC epitope tag, and the $A R R-M Y C$ fusion genes were placed under the control of a CaMV $35 \mathrm{~S}$ promoter. The resulting constructs were transformed into wild-type (Columbia-0, Col-0) plants. For each construct, at least five independent transgenic lines were initially analyzed in the T2 generation, and then at least two representa- 
tive lines (homozygous T3 or T4 plants) were used in all experiments described below. Representative results obtained from one set of transgenic lines are presented in this report and a part of results obtained from an independent set of transgenic lines are included in the Supplementary Data. In these transgenic lines, respective transgenes showed a variety of expression levels as revealed by RT-PCR (Figure 1A). For conciseness, hereafter we refer to these transgenic lines as $A R R$-overexpression or $A R R$-OX lines.

\section{Distinctive phenotypes of type-A ARR-OX transgenic plants}

Under normal growth conditions, $A R R$-OX transgenic plants showed a variety of phenotypes. The primary root length of ARR3-OX, ARR4-OX, ARR5-OX, ARR16$\mathrm{OX}$ and $A R R 17-\mathrm{OX}$ plants was significantly longer than that of wild type $(P<0.01)$, and other transgenic plants showed a marginal phenotype (Figures 1B and 1C; Supplementary information, Figure S1A). In these transgenic lines, ARR3-OX, ARR16-OX and ARR17-OX showed relatively stronger phenotype in primary root growth, with root length over 30\% (ARR3-OX) and 20\% (ARR16$\mathrm{OX}$ and $A R R 17-\mathrm{OX})$ longer than that of wild-type plants (Figure 1C).

Overexpression of type-A $A R R$ genes substantially affected lateral root development, as most $A R R$-OX plants (except ARR4-OX, ARR5-OX and ARR7-OX) had more lateral roots than wild-type plants $(P<0.01)$ (Figure 2). Again, the ARR3-OX transgenic plants showed the stron-
A
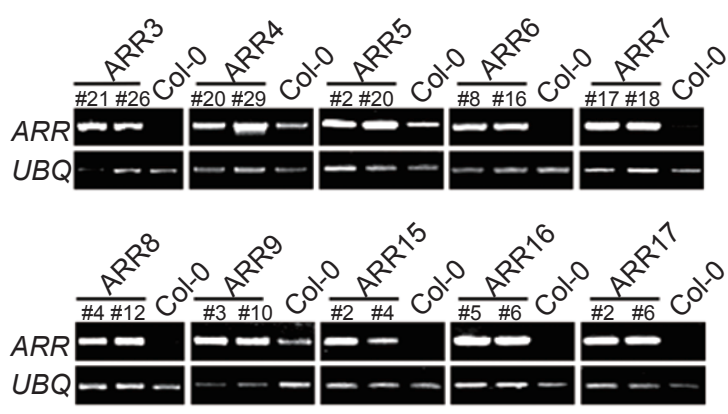

C

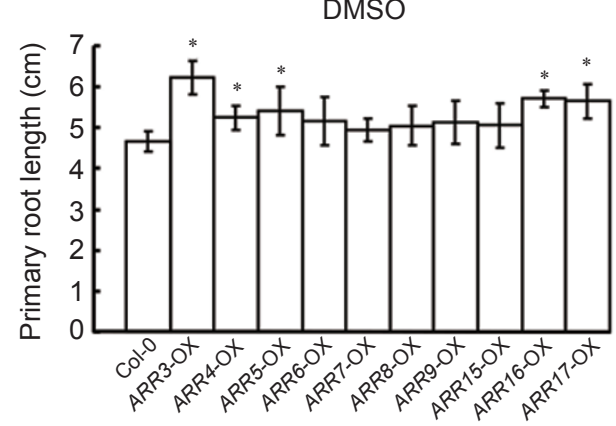

B

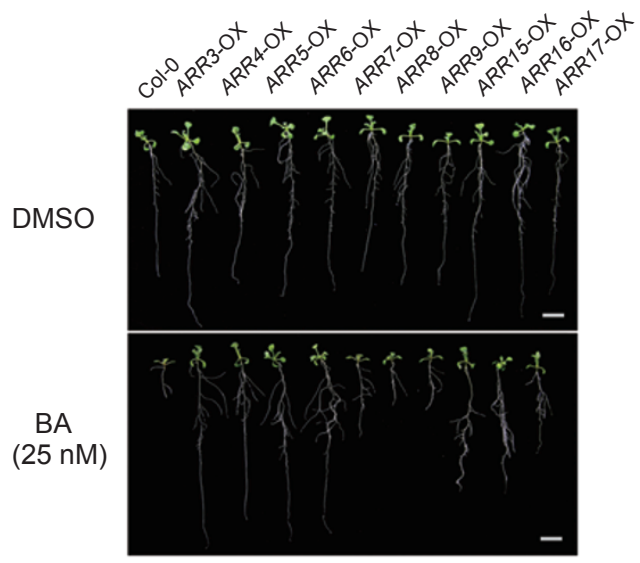

$\mathrm{BA}(50 \mathrm{nM})$

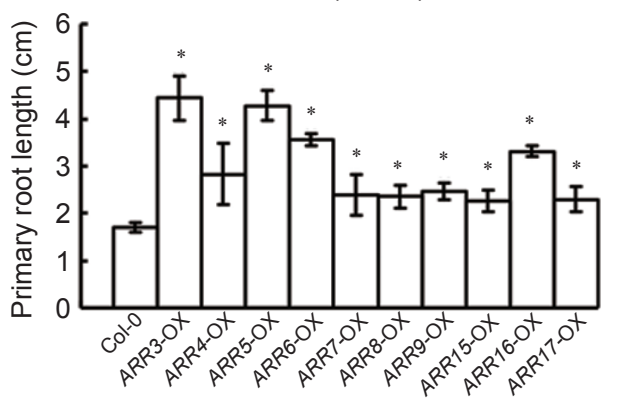

Figure 1 Characterization of type-A $A R R$-overexpression (ARR-OX) transgenic plants. (A) Expression of type-A ARR genes analyzed by RT-PCR in wild-type (Col-0) and $A R R$-OX plants. At least five independent transgenic lines for each transgene were analyzed, and results of two representative lines are shown. Transgenic line numbers are indicated on the top of the panel. Ubiquitin (UBQ) was used as an internal control. UBQ and ARR genes were amplified for 24 and 26 cycles, respectively. Primers used in PCR are the same pairs for the cloning experiment (see Supplementary information, Table S1), which recognize both endogenous and transgenic transcripts of each gene. Similar results were obtained by using transgenespecific primers (data not shown). (B) A total of 2-week-old wild-type (Col-0) and ARR-OX transgenic seedlings germinated and grown on 1/2 MS medium containing dimethyl sulfoxide (DMSO) or $25 \mathrm{nM}$ benzyladenine (BA). Bar, $1 \mathrm{~cm}$. (C) Primary root length of wild-type (Col-0) and ARR-OX seedlings grown on MS agar plates for 8 days. (D) Primary root length of wildtype (Col-0) and $A R R$-OX seedlings (8-day old) in the presence of $50 \mathrm{nM}$ benzyladenine (BA). Asterisks in panels (C) and (D) indicate statistically significant differences compared with wild type (ANOVA analysis, $P<0.01$ ). 


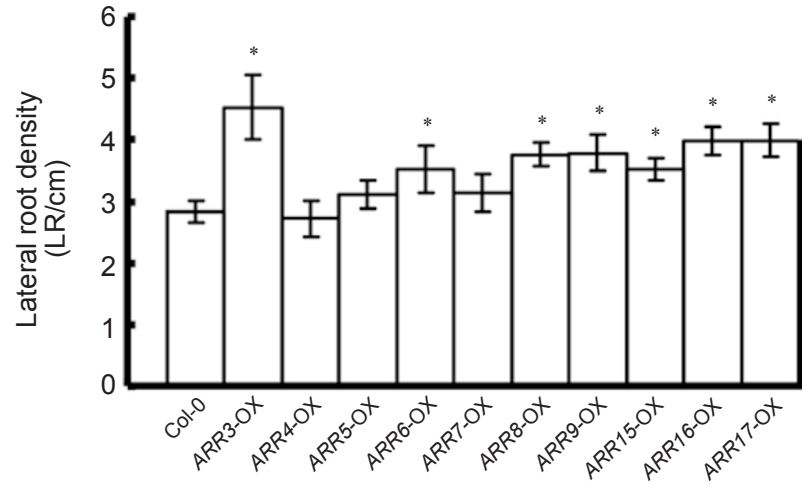

Figure 2 Lateral root development in type-A ARR-OX plants. Seedlings were germinated and grown on MS agar plates for 10 days. The lateral root (LR) number was counted and converted into the number of LR per centimeter of the primary root, as shown in the graph. The data shown are mean values obtained from three experiments. Error bars represent SD $(n>20)$. Asterisks indicate statistically significant differences compared with wild type (ANOVA analysis, $P<0.01$ ).

gest phenotype with $60 \%$ more lateral roots than wildtype plants (Figure 2). ARR16-OX and ARR17-OX also had substantially more lateral roots $(\sim 40 \%)$ than wildtype plants (Figure 2). Notably, whereas ARR4-OX and $A R R 5$-OX showed a similar phenotype as wild-type plants in lateral root development, both transgenic lines however had longer primary roots (Figures $1 \mathrm{C}$ and 2). Collectively, these results suggest that type-A $A R R$ genes have overlapped functions in root development, but may employ distinctive mechanisms to regulate development of primary roots and lateral roots.

Most ARR-OX plants, except ARR5-OX and ARR15$O X$, flowered earlier than wild type when grown under continuous white light $(P<0.01)$ (Figure 3 and Supplementary information, Figure S2). Similar observations were made in the previous studies on ARR4-OX, ARR8$\mathrm{OX}$ and $A R R 7-\mathrm{OX}$ transgenic plants [43, 45]. In contrast to their dominant roles in root development, ARR3-OX and $A R R 16-\mathrm{OX}$ transgenics showed a moderate phenotype in the flowering time. Instead, ARR4-OX and ARR7OX, which showed a weak or a wild-type-like phenotype in root development (see above), had a more profound effect on the control of the flowering time. Among all the tested transgenics, ARR4-OX plants showed the strongest early flowering phenotype (Figure 3 and Supplementary information, Figure S2), consistent with its role in the regulation of light signaling $[37,38,40]$. The $A R R 17-\mathrm{OX}$ transgenic plants showed relatively strong phenotype in both root development and the flowering time (Figures 1B, 2 and 3). Therefore, as summarized in Table 1, the function of type-A $A R R$ genes is partially overlapped and, on the other hand, also shows a gene-specific pattern during plant growth and development (see Discussion).

Type-A ARR-OX transgenic plants show reduced sensitivity to cytokinin in root growth

We examined the sensitivity of $A R R$-OX transgenic plants to cytokinin in several assays, focusing on the regulatory roles of these response regulators in cytokininmediated growth and development. External application of cytokinin inhibits primary root elongation. When treated with cytokinin, all $A R R$-OX transgenic plants had longer primary roots than wild-type plants (Figure 1D and Supplementary information, Figure S1B). Under our assay conditions, treatment with 0.05 and $5 \mu \mathrm{M}$ benzyladenine (BA) caused more than $50 \%$ and $85 \%$ inhibition of primary root elongation in wild-type plants, respectively (Figure 4 and Supplementary information, Figure S3). Primary root elongation of $A R R$-OX transgenic plants showed reduced sensitivity to cytokinin at varying degrees (Figures 1D and 4; Supplementary information, Figure S3). In the presence of $0.05 \mu \mathrm{M}$ BA, the root length of all the transgenic lines was longer than that of wild type (Figure 1D; $P<0.01$ ). When treated with different concentrations of cytokinin, ARR3-OX, ARR5-OX, ARR6-OX and ARR16-OX transgenic plants had longer primary roots than other $A R R$-OX transgenic lines (Figure 4 and Supplementary information, Figure S3). Of these four lines, ARR5-OX showed the strongest phenotype, although it had a relatively weak phenotype under normal growth conditions, especially in lateral root development and the flowering time (Figures 2 and 3).

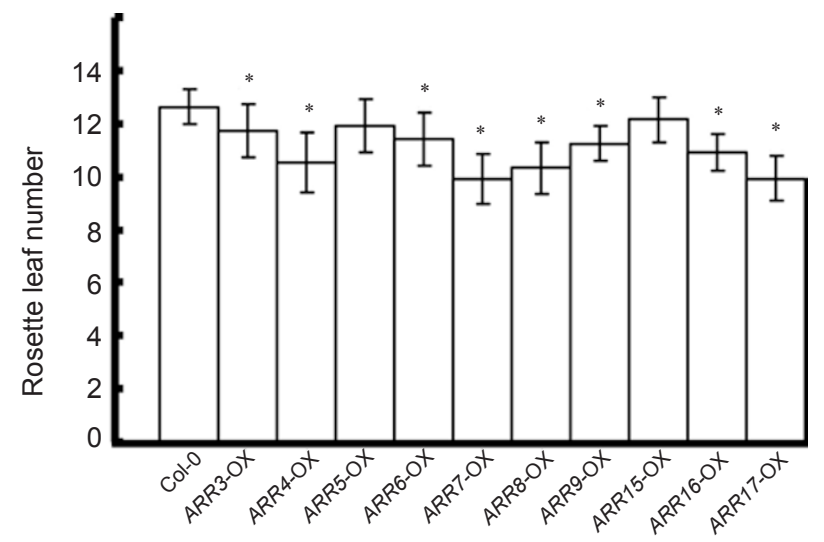

Figure 3 The flowering phenotype of the type-A $A R R-O X$ plants. The flowering time of wild-type and $A R R$-OX plants grown under continuous white light was measured by counting rosette leaf number at bolting. The experiment was repeated thrice and the average values are shown. Error bars represent SD $(n>30)$. Asterisks indicate statistically significant differences compared with wild-type plants (ANOVA analysis, $P<0.01$ ). 

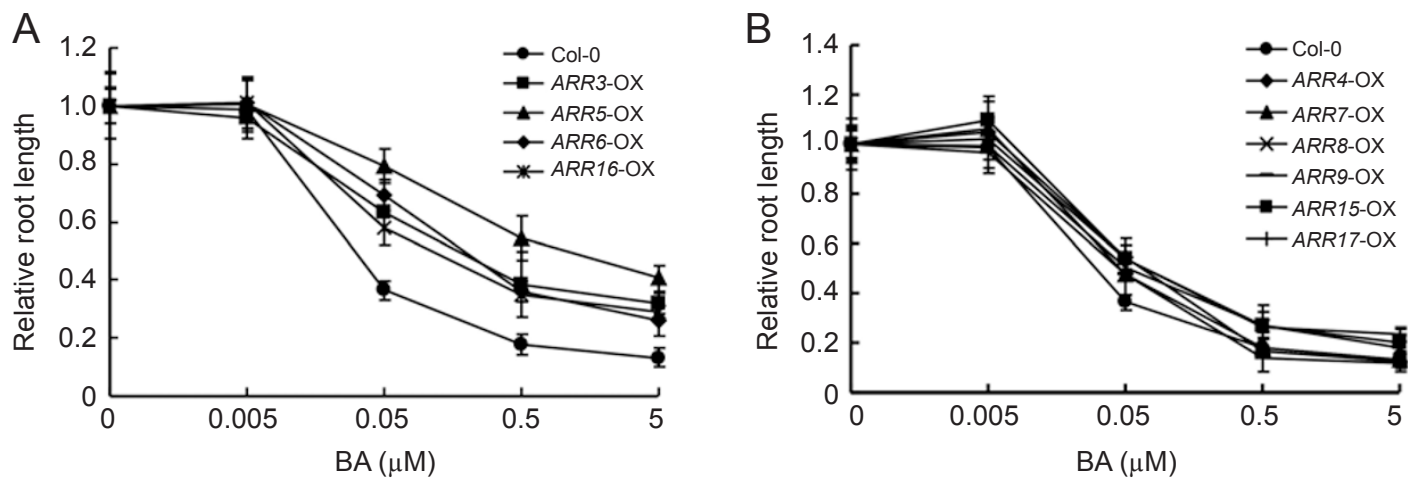

Figure 4 Reduced cytokinin sensitivity of type-A $A R R$-OX plants in the inhibition of primary root elongation. Seedlings were germinated and grown vertically on MS agar plates containing various concentrations of DMSO or BA as indicated. Primary root length was measured 8 days post germination. The data shown are mean values obtained from three experiments. Error bars represent SD $(n>20)$. (A) Relative length of primary roots of $A R R 3,5,6,16-O X$ seedlings germinated and grown in the presence of different concentrations of BA as indicated. Relative length of primary roots of respective plants in the absence of $\mathrm{BA}$ are set as 1.0. These transgenic lines show a stronger phenotype. (B) Relative length of primary roots of $A R R 4,7,8,9$, $15,17-O X$ seedlings germinated and grown in the presence of different concentrations of BA, as indicated. Relative length of primary roots of respective plants in the absence of BA are set as 1.0. These transgenic lines show a response similar to that of wild-type plants.

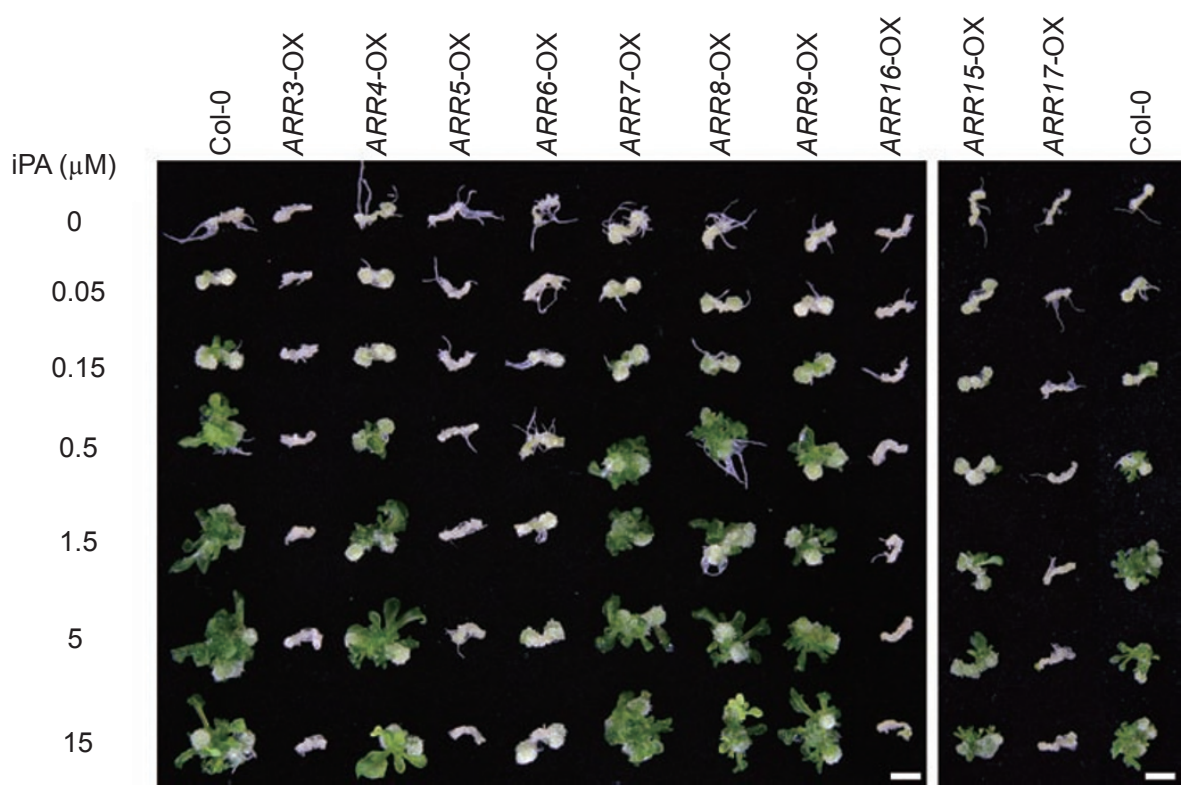

Figure 5 Shoot regeneration capacity of hypocotyl explants of type-A ARR-OX plants. Hypocotyl explants derived from 4-dayold seedlings germinated and grown on GM media under dim light were used for the shoot regeneration assay. After culturing for 4 weeks on the regeneration medium containing different concentrations of cytokinin (iPA) as indicated at the left side, representative calli or shoots were photographed. At least 60 explants derived from seedlings of a given transgenic line were used in each experiment. The experiment was repeated thrice and similar results were obtained. Bar, $5 \mathrm{~mm}$.

Cytokinin has been known to regulate root xylem development by promoting protoxylem differentiation $[47$, 48]. However, no significant effects on root xylem development were observed in all tested $A R R$-OX transgenic plants in the absence or the presence of cytokinin (see Supplementary information, Figure S4 for examples). Moreover, an arr3, 4, 5, 6, 8, 9 hextuple mutant [42] did not show detectable abnormalities in root xylem de- 
velopment in the absence or the presence of cytokinin (Supplementary information, Figure S4).

Cytokinin-induced shoot regeneration capacity is compromised in type-A ARR-OX transgenic plants

In vitro shoot formation is a highly specific assay for the cytokinin response $[12,49]$. We tested the response of hypocotyl explants derived from $A R R$-OX plants to cytokinin in a shoot regeneration assay. Compared with that of wild type, hypocotyl explants of ARR3-OX, ARR5-OX, ARR6-OX, ARR16-OX and ARR17-OX transgenics were nearly insensitive to cytokinin (2-isopente-
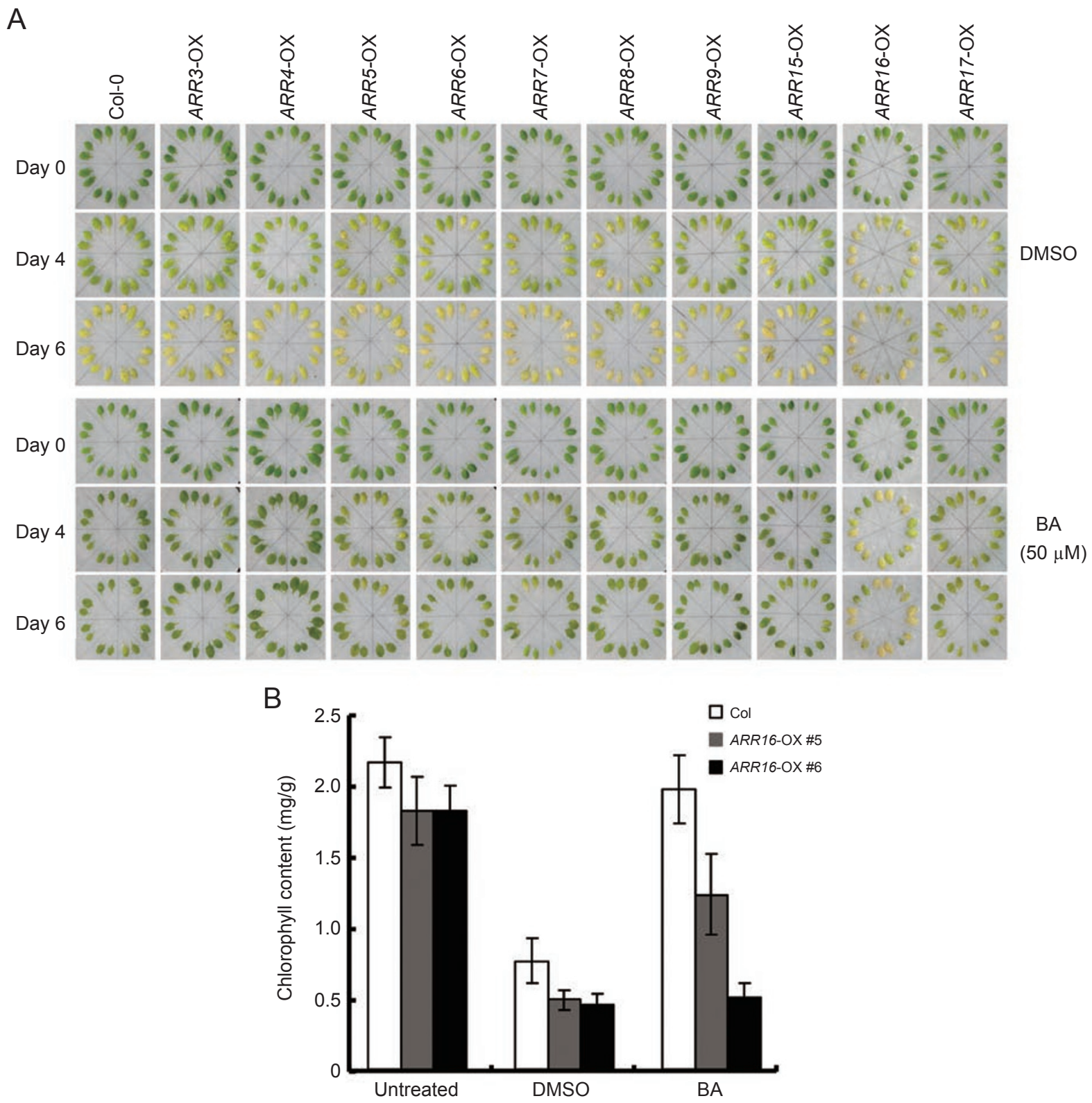

Figure 6 Dark-induced leaf senescence of type-A ARR-overexpression plants. (A) The third and fourth true leaves derived from 3-week-old seedlings were placed on wet filter papers containing $50 \mu \mathrm{M}$ BA or DMSO as indicated. The samples were cultured in the dark at $22{ }^{\circ} \mathrm{C}$ for 0,4 or 6 days. Leaves were collected from at least eight seedlings in each sample. The experiment was repeated thrice and similar results were obtained. ARR16-OX leaves shown in the panel were collected from line \#6. (B) Measurement of the chlorophyll contents in leaves collected from wild-type (Col-0) and ARR16-OX (lines \#5 and \#6) plants. Leaves were treated with DMSO or BA for 4 days as described in panel (A). Untreated: samples prepared from leaves at day 0 . Data presented were mean values from three independent experiments. Bars denote standard deviation. 
nyladenine (iPA)), incapable of forming green calli or shoots even under relatively high concentrations of iPA $(15 \mu \mathrm{M})$ (Figure 5 and Supplementary information, Figure S5). Under the assay condition, ARR15-OX explants showed slightly reduced sensitivity to iPA, whereas other $A R R$-OX explants (4, 7, 8 and 9) displayed a similar response to iPA as wild type (Figure 5 and Supplementary information, Figure S5).

\section{Dark-induced leaf senescence in type-A ARR-OX plants}

Cytokinin is able to delay senescence of detached leaves in the dark. We performed a dark-induced leaf senescence assay of $A R R$-OX transgenic plants to evaluate possible roles of these genes in cytokinin-regulated leaf senescence. In the absence or the presence of cytokinin, leaves collected from most $A R R$-OX transgenic plants showed a phenotype similar to that of wild type (ARR3-
OX, ARR4-OX and ARR9-OX) or a marginally altered phenotype (ARR5-OX, ARR6-OX, ARR7-OX, ARR8OX, ARR15-OX and ARR17-OX) (Figure 6A). However, ARR16-OX leaves displayed an early senescence phenotype, and had a substantially reduced response to cytokinin in the dark-induced leaf senescence assay (Figure 6A). To quantitatively analyze the senescence phenotype of ARR 16-OX leaves, we measured the chlorophyll level in wild-type and the transgenic leaves (Figure 6B). ARR16-OX leaves contained a slightly lower level of chlorophylls than that of wild type. The dark treatment caused a more rapid loss of chlorophylls in ARR16-OX leaves. When treated with cytokinin, whereas wild-type leaves retained chlorophylls at a similar level as the untreated leaves, ARR16-OX leaves showed substantially reduced response to cytokinin (Figure 6B). This result suggests that ARR16 may be involved in the regulation
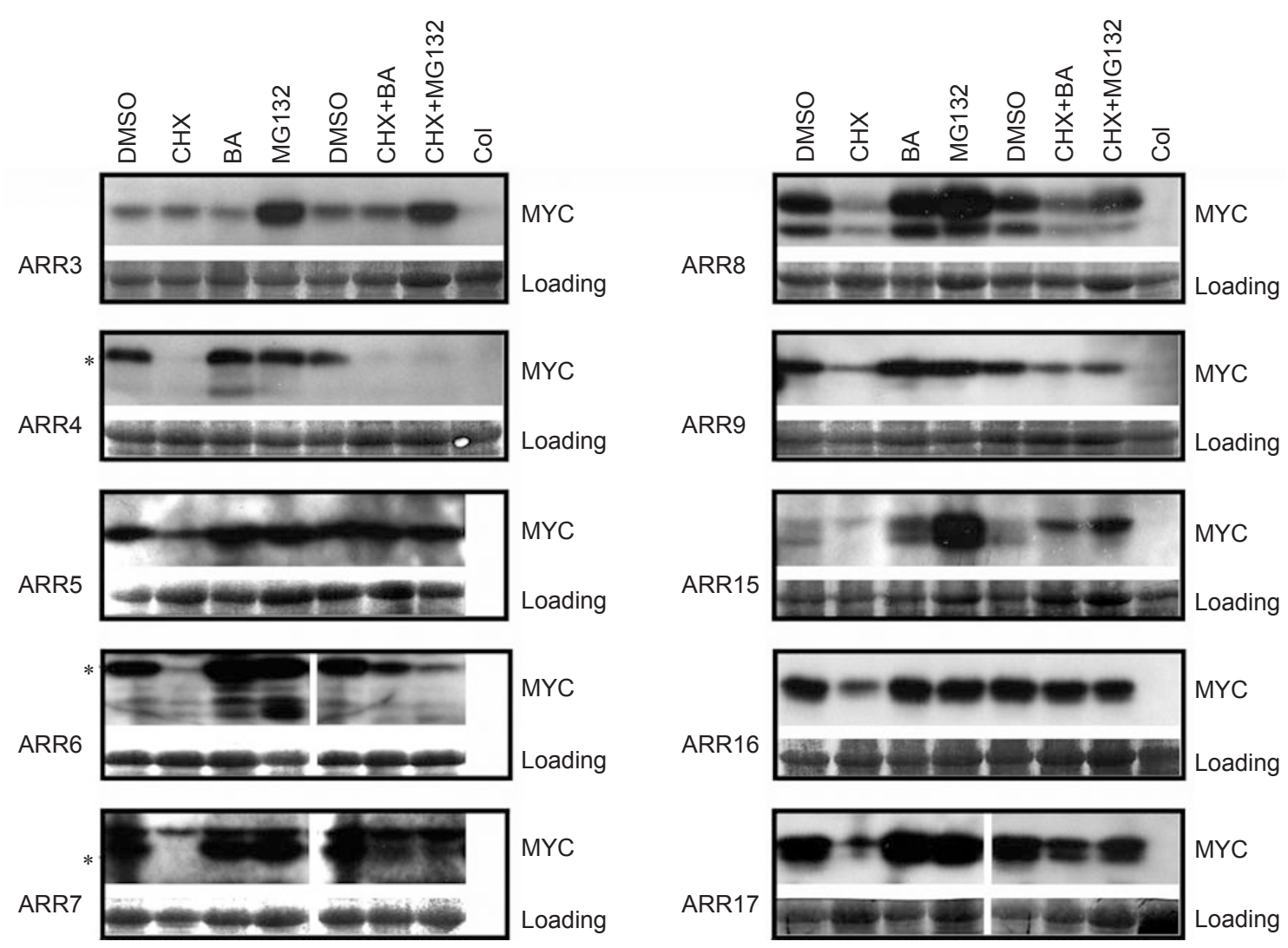

Figure 7 The accumulation of ARR-MYC proteins is regulated by cytokinin and MG132. Seedlings (2-week-old) were treated with different chemicals (indicated on the top of the panel) for $3 \mathrm{~h}$ in $1 / 2 \mathrm{MS}$ liquid medium. Extracts equivalent approximately 15-60 $\mu \mathrm{g}$ of proteins were used for SDS-PAGE and subsequent western blot analysis using an anti-MYC monoclonal antibody. The blot stained with Ponceau $S$ was used as loading controls (bottom of each blot). Asterisks in ARR4, ARR6 and ARR7 blots denote bands that are presumably specific to the transgenes. In ARR17, two close-migrated bands were detected. In ARR6, ARR7 and ARR17 panels, data presented are derived from the same blots. The experiment was repeated at least thrice and similar results were obtained. Representative blots are shown. DMSO: dimethyl sulfoxide; $\mathrm{CHX}$ : cyclohexi-

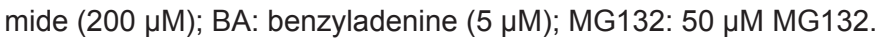


of cytokinin-controlled leaf senescence.

\section{Analysis of type-A ARR-MYC proteins in transgenic plants}

Expression of type-A ARR genes has been shown to be rapidly induced by cytokinin which does not require de novo protein synthesis $[35,36]$. Constitutive expression of type-A $A R R$ genes by a $35 \mathrm{~S}$ promoter therefore allows the study of possible posttranslational regulation of ARR proteins. We analyzed individual ARR-MYC proteins by western blot using an anti-MYC monoclonal antibody. Compared with the relatively constant level of mRNA as revealed by RT-PCR (see Figure 1A), ARR-MYC protein levels substantially varied in different transgenic plants, implying the involvement of possible translational or posttranslational controls (see below).

In most cases, we have been able to detect single protein bands that were specific to related transgenic plants and approximately correlated to the predicted molecular weights (MW). In ARR4-OX transgenic lines, however, a protein band with an apparent MW over $50 \mathrm{kD}$, instead of the predicted MW of $\sim 37 \mathrm{kD}$, was specifically detected (Supplementary information, Figure S6). A similar observation has also been made in a previous study (see Figure 2B, [46]). Because no other specific band was detected in ARR4-OX transgenic plants, we followed To et al. [46] to presume the $\sim 50-\mathrm{kD}$ band as ARR4-MYC. In addition, we noticed that two protein bands were reproducibly detected in multiple $A R R 8-\mathrm{OX}, A R R 15-\mathrm{OX}$ and ARR17-OX transgenic lines (Figure 7 and Supplementary information, Figure S7). Both bands appeared to be specific to the respective transgenic lines, because similar bands were not detected by the anti-MYC monoclonal antibody in wild-type and other transgenic plants. The lower bands in the ARR8-MYC and ARR15 blots appeared to be correlated to the predicted MW of the respective fusion proteins (approximately 34 and $31 \mathrm{kD}$, respectively). We speculate that some of these bands may be caused by posttranslational modifications of these proteins, albeit the precise biochemical mechanism of the possible modifications remains to be elucidated.

The accumulation of type- $A$ ARR-MYC proteins is regulated by cytokinin and the proteasome pathway

We found that cytokinin was able to increase the accumulation of ARR8-MYC, ARR15-MYC, ARR16-MYC and ARR17-MYC fusion proteins (Figure 7 and Supplementary information, Figure S7). Stability of ARR5MYC, ARR6-MYC and ARR7-MYC proteins was also increased by cytokinin, consistent with the observation in a previous study [46]. The elevated protein level stimulated by cytokinin in these transgenic lines could be caused by an increased translation rate or increased protein stability, or by a combination of both effects. To distinguish these possibilities, we treated the transgenic plants with the protein synthesis inhibitor cycloheximide (CHX) in the absence or the presence of cytokinin. The CHX treatment significantly reduced the accumulation of ARR8-MYC, ARR15-MYC, ARR16-MYC and ARR17MYC proteins, indicating that stability of these proteins is regulated. In the presence of $\mathrm{CHX}$, the reduced protein level of ARR16-MYC and ARR17-MYC could be partially rescued by cytokinin (Figure 7 and Supplementary information, Figure S7; lanes $\mathrm{CHX}$ vs lanes $\mathrm{CHX}+\mathrm{BA}$ ). These results suggest that cytokinin is capable of stabilizing ARR16-MYC and ARR17-MYC proteins.

ARR8-MYC and ARR15-MYC proteins showed a complicated pattern. In the presence of CHX, the reduced level of the lower bands of ARR8-MYC and ARR15MYC (see above for explanation) could not be rescued by cytokinin. However, the accumulation of upper bands appeared to be increased upon cytokinin treatment (Figure 7 and Supplementary information, Figure S7; lanes CHX vs lanes $\mathrm{CHX}+\mathrm{BA}$ ). This result suggests that cytokinin may partially stabilize ARR8-MYC and ARR15-MYC proteins.

ARR3-MYC protein did not appear to be regulated by cytokinin (Figure 7 and Supplementary information, Figure S7), showing a phenotype similar to that of ARR4MYC and ARR9-MYC. Moreover, the ARR3-MYC level was not significantly altered by $\mathrm{CHX}$, indicating that the protein has a relatively longer half-life.

To ask if stability of ARR-MYC proteins is also regulated by the proteasome pathway, we treated the transgenic plants with MG132, a specific proteasomal inhibitor, in the absence or the presence of CHX. We found that ARR3-MYC, ARR5-MYC, ARR7-MYC, ARR16MYC and ARR17-MYC proteins could be stabilized by MG132, suggesting that stability of these proteins is subjected to regulation by the proteasome pathway (Figure 7 and Supplementary information, Figure S7). Similar to that of the cytokinin treatment, lower bands of ARR8MYC and ARR15-MYC did not appear to be affected by MG132. However, the upper bands of these two proteins were substantially upregulated by MG132 (Figure 7 and Supplementary information, Figure S7). Levels of ARR4-MYC, ARR6-MYC and ARR9-MYC proteins were only marginally altered by MG132, suggesting that these three proteins may not be significantly regulated by the proteasome pathway. Collectively, these results suggest that stability of ARR proteins is regulated by distinctive mechanisms involving cytokinin regulation and/ or proteasomal degradation or both.

Phenotypes of type-A ARR-OX transgenic plants and 
stability regulation of ARR-MYC proteins are summarized in Table 1.

\section{Discussion}

In Arabidopsis, cytokinin signaling is mediated by a TCS consisting of AHKs, AHPs and ARRs. As an output of the signaling pathway, ARR proteins are the main effectors, by which cytokinin executes its various functions during plant growth and development. Owing to functional redundancy, it is technically difficult to study the function of type-A $A R R$ genes by using loss-of-function mutants [27, 28, 42]. In fact, our current understanding of the type-A $A R R$ functions was largely obtained from overexpression studies of several type-A $A R R$ genes $[18,43-46]$. In this study, we performed a genome-wide comparative analysis on the function of all type-A $A R R$ genes, aimed at gaining comprehensive and new insights into their functions and regulatory mechanisms.

\section{Redundant and diverse functions of type-A ARR genes in plant growth and development}

Most key components in the cytokinin signaling pathway function redundantly, and this redundancy is particularly apparent in genes encoding for receptors, phosphotransfer proteins and response regulators. However, these components also appear to function distinctively in plant growth and development. It has been proposed that different receptors or receptor combinations contribute to distinctive aspects of plant growth and development [11]. For instance, CRE1/WOL/AHK4 is predominantly involved in root xylem specification [47, 48], whereas AHK3 plays an important role in the regulation of leaf senescence [50]. Likewise, type-A $A R R$ genes may also have overlapped yet distinctive functions in a gene-specific manner to regulate different aspects of plant growth and development. In agreement with this notion, it has been shown that individual or combinations of type-A $A R R$ genes play distinctive roles in the regulation of red light signaling, the circadian clock, meristem development and stem-cell specification (see Introduction for detailed references).

The gene-specific action mode of the functionally redundant type-A $A R R$ genes perhaps could be best exemplified by $A R R 3$ and $A R R 4$. These two genes encode two structurally closely-related proteins that function redundantly to regulate the circadian period in a cytokininindependent manner [37]. ARR4 physically interacts with phytochrome B to regulate red light signaling [40], and possibly circadian clock oscillation as well [38]. However, whereas $A R R 4-\mathrm{OX}$ shows a marginally altered response to cytokinin, $A R R 3-\mathrm{OX}$ displays substantially reduced response to this phytohormone. Intriguingly, neither cytokinin nor MG132 appears to be able to af-

Table 1 Summary of type-A ARR-OX transgenic phenotypes

\begin{tabular}{|c|c|c|c|c|c|c|c|}
\hline \multirow{3}{*}{ Genotype } & \multicolumn{3}{|c|}{ Growth Phenotype $^{\mathrm{a}}$} & \multirow{3}{*}{ Leaf } & \multirow{3}{*}{ Shoot } & \multicolumn{2}{|c|}{ Protein level $^{\mathrm{b}}$} \\
\hline & Primary & Lateral & Flowering & & & BA & MG132 \\
\hline & Root & Root & & & & & \\
\hline ARR3-OX & Significant & Significant & Significant & WT & Strong & - & + \\
\hline ARR4-OX & Significant & WT & Significant & WT & WT & - & - \\
\hline ARR6-OX & Weak & Significant & Significant & WT & Strong & + & - \\
\hline ARR7-OX & Weak & Weak & Significant & WT & WT & + & + \\
\hline ARR8-OX & Weak & Significant & Significant & WT & WT & $+^{\mathrm{c}}$ & $+^{\mathrm{c}}$ \\
\hline ARR9-OX & Weak & Significant & Significant & WT & WT & - & - \\
\hline ARR15-OX & Weak & Significant & WT & WT & Weak & $+^{c}$ & $+^{\mathrm{c}}$ \\
\hline
\end{tabular}

${ }^{\mathrm{a}}$ Data presented on primary root elongation, lateral root number and the flowering time are obtained under the normal growth condition. Shoot formation refers to the cytokinin sensitivity of hypocotyl explants derived from ARR-OX transgenics. The relative strength of the phenotype is indicated as Significant (statistically significant), Weak (marginally altered), WT (wild-type-like) or Strong (nearly insensitive; for the shoot formation assay only).

${ }^{\mathrm{b}}$ Protein level refers to the accumulation of ARR-MYC proteins regulated (+) or not-regulated (-) by benzyladenine (BA) and MG132, respectively. ${ }^{c}$ Two bands were detected by an anti-MYC antibody in ARR8-OX and ARR15-OX transgenic plants. Both bands appear to be specific to respective transgenes. Treatment by cytokinin or MG132 does not have significant effects on the accumulation of the lower bands in both cases, but does cause an altered level of the upper bands (see Figure 7 and text for more details). 
fect the ARR4-MYC protein level, whereas MG132 can substantially increase the ARR3-MYC protein level (see also below). Collectively, these observations indicate that ARR3 and ARR4, function redundantly in the activity associated with circadian clock oscillation, but execute different functions in other aspects of plant growth and development, which is consistent with the possible involvement of distinctive regulatory mechanisms. A similar pattern can also be noticed for ARR5 and ARR6, which fall into the same phylogenetic branch [51]. Overexpression of these two genes, however, causes nearly opposite phenotypes in primary root growth, lateral root development and the flowering time, as well as in the regulation of protein levels by the proteasome pathway (Table 1). These results provide an additional example of diverse functions and regulatory mechanisms of type-A ARR proteins.

It is interesting to note that $A R R 16$, in addition to its role in root growth and shoot regeneration, also affects leaf senescence. It appears that overexpression of $A R R 16$ displays a phenotype stronger than any other type-A $A R R$-OX transgenics thus far analyzed, suggestive of its important roles in plant growth and development. Despite the relatively strong phenotype of ARR16-OX, an arr 16 loss-of-function mutant does not have detectable phenotype under normal growth conditions and shows a normal response to cytokinin (Ren and Zuo, unpublished data), providing an additional line of evidence of functional redundancy of type-A $A R R$ genes.

A major physiological role of cytokinin is to regulate root xylem development. Mutations in $A H K, A H P$ and type-B $A R R$ genes cause reduced protoxylem differentiation [16, 28-30, 47, 48]. However, we could not observe detectable abnormalities in root xylem development in all $A R R$-OX transgenic lines that were grown in the absence or the presence of cytokinin. One possibility is that overexpression of type-A $A R R$ genes under our experimental conditions is unable to attenuate cytokinin signaling to a sufficiently low level, at which root xylem differentiation may be affected. Alternatively, root xylem development is less sensitive to the intracellular concentrations of individual type-A ARR proteins, owing to functional redundancy or buffering capacity of this family of proteins. This view is supported by the observation that an arr3, $4,5,6,8,9$ hextuple mutant did not show abnormal development of root xylem, although other developmental defects were detectable in the mutant.

Regulation of stability of type-A ARR proteins by cytokinin and proteasome machinery

The discovery of type-A $A R R$ genes was based on their cytokinin-inducibility in the absence of de novo protein synthesis [35, 36]. Considering the complexity of cytokinin signaling, it is reasonable to predict additional mechanisms involved in the regulation of the activity of this class of proteins. Recent studies demonstrated that cytokinin is indeed able to stabilize a subset of type-A ARR proteins, including ARR5, ARR6 and ARR7 [46]. Similarly, we found that stability of other type-A proteins, including ARR16 and ARR17 (possibly ARR8 and ARR15), is also regulated by cytokinin. More importantly, our results revealed an additional mechanism involved in the regulation of ARR proteins. The accumulation of several ARR-MYC proteins can be substantially increased by MG132 in the absence or the presence of de novo protein synthesis, suggesting involvement of the proteasomal degradation in the regulation of these proteins.

It has been noticed that the overall design and action of cytokinin signaling are similar to that of auxin signaling $[42,51]$. In the auxin signaling pathway, AUX/ IAA proteins, encoded by auxin-inducible genes, act to repress the activity of a class of transcription factors, termed as auxin response factors (ARFs). Auxin induces the degradation of the AUX/IAA repressors, thereby derepressing ARFs and consequently activating the signaling pathway [52]. Analogously, type-A ARRs repress the activity of type-B ARRs, also a class of transcription factors, and the repression is relieved by cytokinin [4, $18,51,53]$. An attractive model is that type-A ARR proteins, similar to AUX/IAA proteins, are subjected to the regulation by proteasomal degradation, and destruction of the repressive type-A ARR proteins thus leads to the activation of type-B ARRs $[42,51]$. Consistent with this model, mutations in COP10/FUS9/CIN4 and RPN12, which encode subunits of the $26 \mathrm{~S}$ proteasome, lead to insensitivity to cytokinin $[54,55]$, and the reporter activity of an ARR7-luciferase fusion gene is enhanced by MG132 in transiently transformed protoplasts [56]. The observation that the accumulation of a subset of type-A ARR proteins is increased by MG132 provides a line of supporting evidence that cytokinin signaling is regulated by a mechanism involving proteasomal degradation of certain type-A ARRs, which may act to derepress type-B ARRs.

It is worthwhile to note that, as implicated from its role in the regulation of red light signaling, the accumulation of ARR4 is induced by white light and red light, but repressed by far-red light in a phytochrome B-dependent manner [40]. Thus, it appears that stability of type-A ARR proteins is regulated by diverse mechanisms, involving at least the cytokinin, light and proteasome pathways. These regulatory mechanisms are further complicated by different combinations of the cytokinin 
and MG132 actions on type-A ARR proteins. As summarized in Table 1, six type-A ARR proteins (ARR5, ARR7, ARR8, ARR15, ARR16 and ARR17) appears to be regulated by both cytokinin and proteasomal degradation. Moreover, whereas ARR3 and ARR6 are sensitive to MG132 and cytokinin, respectively, ARR4 and ARR9 show no response to either reagent. This combinatorial regulatory mechanism likely plays an important role in the control of the ARR activity. Obviously, unraveling the biochemical natures of these regulatory mechanisms, in particular, identification and characterization of E3 ligases specific to type-A ARR proteins, will be crucial toward a better understanding of cytokinin signaling, as well as its roles in plant growth and development.

\section{Materials and Methods}

\section{Plant materials and growth conditions}

Unless specified, the Columbia-0 (Col-0) accession of Arabidopsis was used in this study. The arr 3,4,5,6,8,9 mutant was kindly provided by Dr Joe Kieber [42]. Unless otherwise indicated, plants were grown under continuous white light at $22{ }^{\circ} \mathrm{C}$ in soil or on Murashige-Skoog (MS) [57] medium (1/2 MS salts, $1 \%$ sucrose and $0.8 \%$ agar).

\section{Plasmid construction and generation of transgenic lines}

Total RNA prepared from different tissues of wild-type plants was used as templates to synthesize the first strand of cDNA using oligo-dT as a primer. The reaction was used to PCR-amplify cDNA sequences corresponding to the coding region (lacking stop codons) of type-A ARR genes using PWO DNA polymerase (Roche Diagnostics Hong Kong, Hong Kong, China), and the resulting PCR fragments were cloned into a pGEM-T easy vector (Promega, Madison, WI, USA). All primers used in PCR are listed in Supplementary information, Table S1. After confirmation by DNA sequencing, the ARR fragments were cloned into the XhoI and $E c o$ RI (or $E c o$ RV) sties of a pSK-c-MYC vector, which contained six copies of a MYC epitope followed by an in-frame stop codon. The ARR-MYC fusion genes were then cloned into the $X h o \mathrm{I}$ and SpeI sites of a pBA-0 vector under the control of the CaMV $35 \mathrm{~S}$ promoter carrying a bialaphos-resistance selectable gene. The resulting constructs were transformed into Agrobacterium tumefaciens strain GV3101, which was then used for the transformation of plants (Col-0) by the floral dip method as described [58].

\section{$R N A$ preparation and $R T-P C R$}

Total RNA was extracted from 10-day-old seedlings with the TRIZol reagent (Invitrogen) according to the manufacturer's instructions. RT-PCR was carried out as previously described [59]. The reaction was cycled $24-26$ times at $94{ }^{\circ} \mathrm{C}$ for $30 \mathrm{~s}, 58^{\circ} \mathrm{C}$ for $30 \mathrm{~s}$ and $72{ }^{\circ} \mathrm{C}$ for $1 \mathrm{~min}$.

\section{Analysis of cytokinin responses}

For root elongation inhibition assay, Arabidopsis seedlings were grown vertically on $1 / 2$ MS agar supplemented with appropriate concentrations of BA or dimethyl sulfoxide. Root length was measured 8 days post germination. The flowering time was measured by counting the rosette leaf numbers at bolting. The shoot formation assay [59] and the dark-induced leaf senescence assay $[60]$ were performed as previously described.

\section{Western blot analysis}

Seedlings (2-week-old) were infiltrated in liquid 1/2 MS medium supplemented with, alone or in different combinations (see Figure legends), $200 \mu \mathrm{M}$ CHX, $50 \mu \mathrm{M}$ MG132 or $5 \mu \mathrm{M}$ BA. After the treatment, samples were immediately frozen in liquid nitrogen until the use.

Plant materials were ground in liquid nitrogen into fine powder, and then extracted in $50 \mathrm{mM}$ Tris ( $\mathrm{pH} 7.5), 150 \mathrm{mM} \mathrm{NaCl}, 10 \mathrm{mM}$ $\mathrm{MgCl}_{2}, 1 \times$ complete protease inhibitors (Roche), $0.1 \%$ Nonidet P-40 and $0.5 \% \beta$-mercaptoethanol. After centrifugation in a microcentrifuge for $10 \mathrm{~min}$, the supernants were mixed with equal volume of loading buffer (250 mM Tris, $\mathrm{pH} 6.8,10 \% \mathrm{SDS}, 0.5 \%$ bromophenol blue, $50 \%$ glycerol and $5 \% \beta$-mercaptoethanol). After boiling for $5 \mathrm{~min}$, the sample was separated by $12 \%$ SDS-PAGE and then transferred onto a Westran Clear Signal polyvinylidene difluoride membrane (Whatman). ARR-MYC fusion proteins were detected with an anti-MYC monoclonal antibody (Santa Cruz, CA, USA) as a primary antibody and goat anti-mouse $\operatorname{IgG}$ conjugated with the horseradish peroxidase as a secondary antibody. The signal was detected using the SuperSignal Western Femto Maximun Sensitivity Substrate kit (Pierce Biotechnology), following the manufactures' instructions. Membranes stained by Ponceau S were used as loading controls.

\section{Acknowledgments}

We thank Dr Joe Kieber (University of North Carolina, USA) and the Arabidopsis Biological Resource Center (Ohio State University) for providing seeds. We thank Dr Shuhua Yang (China Agricultural University) for critically reading the manuscript. This work was supported by grants from the National Natural Science Foundation of China (90817107), the Ministry of Science and Technology of China (2007CB948203) and Chinese Academy of Sciences (KSCX2-YW-N-015).

\section{References}

1 Davies PJ. Plant Hormones: Biosythesis, Signal Transduction, Action! 3rd Edition. Dordrecht, The Netherlands: Kluwer Academic Press, 2004.

2 Mok DW, Mok MC. Cytokinin metabolism and action. Annu Rev Plant Physiol Plant Mol Biol 2001; 52:89-118.

3 Hwang I, Chen H-C, Sheen J. Two-component signal transduction pathways in Arabidopsis. Plant Physiol 2002; 129:500-515.

4 Kakimoto T. Perception and signal transduction of cytokinins. Annu Rev Plant Biol 2003; 54:605-627.

5 To JPC, Kieber JJ. Cytokinin signaling: two-components and more. Trends Plant Sci 2008; 13:85-92.

6 Heyl A, Schmülling T. Cytokinin signal perception and transduction. Curr Opin Plant Biol 2003; 6:480-488.

7 Haberer G, Kieber JJ. Cytokinins. New insights into a classic phytohormone. Plant Physiol 2002; 128:354-362.

8 Müller B, Sheen J. Advances in cytokinin signaling. Science 2007; 318:68-69. 
9 Higuchi M, Pischke MS, Mähönen AP, et al. In planta functions of the Arabidopsis cytokinin receptor family. Proc Natl Acad Sci USA 2004; 101:8821-8826.

10 Nishimura C, Ohashi Y, Sato S, et al. Histidine kinase homologs that act as cytokinin receptors possess overlapping functions in the regulation of shoot and root growth in Arabidopsis. Plant Cell 2004; 16:1365-1377.

11 Riefler M, Novak O, Strnad M, Schmülling T. Arabidopsis cytokinin receptor mutants reveal functions in shoot growth, leaf senescence, seed size, germination, root development, and cytokinin metabolism. Plant Cell 2006; 18:40-54.

12 Inoue T, Higuchi M, Hashimoto $\mathrm{Y}$, et al. Identification of CRE1 as a cytokinin receptor from Arabidopsis. Nature 2001; 409:1060-1063.

13 Suzuki T, Miwa K, Ishikawa K, et al. The Arabidopsis sensor His-kinase, AHK4, can respond to cytokinins. Plant Cell Physiol 2001; 42:107-113.

14 Yamada H, Suzuki T, Terada K, et al. The Arabidopsis AHK4 histidine kinase is a cytokinin-binding receptor that transduces cytokinin signals across the membrane. Plant Cell Physiol 2001; 42:1017-1023.

15 Mähönen AP, Higuchi M, Törmäkangas K, et al. Cytokinins regulate a bidirectional phosphorelay network in Arabidopsis. Curr Biol 2006; 16:1116-1122.

16 Hutchison CE, Li J, Argueso C, et al. The Arabidopsis histidine phosphotransfer proteins are redundant positive regulators of cytokinin signaling. Plant Cell 2006; 18:3073-3087.

17 Suzuki T, Sakurai K, Imamura A, et al. Compilation and characterization of histidine-containing phosphotransmitters implicated in His-to-Asp phosphorelay in plants: AHP signal transducers of Arabidopsis thaliana. Biosci Biotechnol Biochem 2000; 64:2486-2489.

18 Hwang I, Sheen J. Two-component circuitry in Arabidopsis cytokinin signal transduction. Nature 2001; 413:383-389.

19 Suzuki T, Imamura A, Ueguchi C, Mizuno T. Histidine-containing phosphotransfer (HPt) signal transducers implicated in His-to-Asp phosphorelay in Arabidopsis. Plant Cell Physiol 1998; 39:1258-1268.

20 Sakai H, Honma T, Aoyama T, et al. ARR1, a transcription factor for genes immediately responsive to cytokinins. Science 2001; 294:1519-1521.

21 Nakamura A, Kakimoto T, Imamura A, et al. Biochemical characterization of a putative cytokinin-responsive Hiskinase, CKI1, from Arabidopsis thaliana. Biosci Biotechnol Biochem 1999; 63:1627-1630.

22 Kiba T, Aoki K, Sakakibara H, Mizuno T. Arabidopsis Response Regulator, ARR22, ectopic expression of which results in phenotypes similar to the wol cytokinin-receptor mutant. Plant Cell Physiol 2004; 45:1063-1077.

23 D'Agostino IB, Deruere J, Kieber JJ. Characterization of the response of the Arabidopsis response regulator gene family to cytokinin. Plant Physiol 2000; 124:1706-1717.

24 D'Agostino IB, Kieber JJ. Phosphorelay signal transduction: the emerging family of plant response regulators. Trends Biochem Sci 1999; 24:452-456.

25 Mason MG, Li J, Mathews DE, Kieber JJ, Schaller GE. Type-B response regulators display overlapping expression patterns in Arabidopsis. Plant Physiol 2004; 135:927-937.

26 Sakai H, Aoyama T, Oka A. Arabidopsis ARR1 and ARR2 response regulators operate as transcriptional activators. Plant $J$ 2000; 24:703-711.

27 Mason MG, Mathews DE, Argyros DA, et al. Multiple type-B response regulators mediate cytokinin signal transduction in Arabidopsis. Plant Cell 2005; 17:3007-3018.

28 Yokoyama A, Yamashino T, Amano Y, et al. Type-B ARR transcription factors, ARR10 and ARR12, are implicated in cytokinin-mediated regulation of protoxylem differentiation in roots of Arabidopsis thaliana. Plant Cell Physiol 2007; 48:84-96.

29 Ishida K, Yamashino T, Yokoyama A, Mizuno T. Three type-B response regulators, ARR1, ARR10 and ARR12, play essential but redundant roles in cytokinin signal transduction throughout the life cycle of Arabidopsis thaliana. Plant Cell Physiol 2008; 49:47-57.

30 Argyros RD, Mathews DE, Chiang YH, et al. Type B response regulators of Arabidopsis play key roles in cytokinin signaling and plant development. Plant Cell 2008; 20:21022116.

31 Heyl A, Ramireddy E, Brenner WG, et al. The transcriptional repressor ARR1-SRDX suppresses pleiotropic cytokinin activities in Arabidopsis. Plant Physiol 2008; 147:1380-1395.

32 Müller B, Sheen J. Cytokinin and auxin interaction in root stem-cell specification during early embryogenesis. Nature 2008; 453:1094-1097.

33 Tajima Y, Imamura A, Kiba T, et al. Comparative studies on the type-B response regulators revealing their distinctive properties in the His-to-Asp phosphorelay signal transduction of Arabidopsis thaliana. Plant Cell Physiol 2004; 45:28-39.

34 Imamura A, Kiba T, Tajima Y, Yamashino T, Mizuno T. In vivo and in vitro characterization of the ARR11 response regulator implicated in the His-to-Asp phosphorelay signal transduction in Arabidopsis thaliana. Plant Cell Physiol 2003; 44:122-131.

35 Brandstatter I, Kieber JJ. Two genes with similarity to bacterial response regulators are rapidly and specifically induced by cytokinin in Arabidopsis. Plant Cell 1998; 10:1009-1019.

36 Imamura A, Hanaki N, Umeda $\mathrm{H}$, et al. Response regulators implicated in His-to-Asp phosphotransfer signaling in Arabidopsis. Proc Natl Acad Sci USA 1998; 95:2691-2696.

37 Salome PA, To JPC, Kieber JJ, McClung CR. Arabidopsis response regulators ARR3 and ARR4 play cytokinin-independent roles in the control of circadian period. Plant Cell 2006; 18:55-69.

38 Zheng B, Deng Y, Mu J, et al. Cytokinin affects circadianclock oscillation in a phytochrome B- and Arabidopsis Response Regulator 4-dependent manner. Physiol Plant 2006; 127:277-292.

39 Ishida K, Yamashino T, Mizuno T. Expression of the cytokinin-induced type-A response regulator gene $A R R 9$ is regulated by the circadian clock in Arabidopsis thaliana. Biosci Biotechnol Biochem 2008; 72:3025-3029.

40 Sweere U, Eichenberg K, Lohrmann J, et al. Interaction of the response regulator ARR4 with phytochrome B in modulating red light signaling. Science 2001; 294:1108-1111.

41 Leibfried A, To JP, Busch W, et al. WUSCHEL controls meristem function by direct regulation of cytokinin-inducible response regulators. Nature 2005; 438:1172-1175.

42 To JPC, Haberer G, Ferreira FJ, et al. Type-A Arabidopsis re- 
sponse regulators are partially redundant negative regulators of cytokinin signaling. Plant Cell 2004; 16:658-671.

43 Lee DJ, Park JY, Ku SJ, et al. Genome-wide expression profiling of ARABIDOPSIS RESPONSE REGULATOR 7 (ARR7) overexpression in cytokinin response. Mol Genet Genomics 2007; 277:115-137.

44 Kiba T, Yamada H, Sato S, et al. The type-A response regulator, ARR15, acts as a negative regulator in the cytokininmediated signal transduction in Arabidopsis thaliana. Plant Cell Physiol 2003; 44:868-874.

45 Osakabe Y, Miyata S, Urao T, et al. Overexpression of Arabidopsis response regulators, ARR4/ATRR1/IBC7 and ARR8/ $A T R R 3$, alters cytokinin responses differentially in the shoot and in callus formation. Biochem Biophys Res Commun 2002; 293:806-815.

46 To JPC, Deruere J, Maxwell BB, et al. Cytokinin regulates type-A Arabidopsis response regulator activity and protein stability via two-component phosphorelay. Plant Cell 2007; 19:3901-3914.

47 Mähönen AP, Bonke M, Kauppinen L, et al. A novel twocomponent hybrid molecule regulates vascular morphogenesis of the Arabidopsis root. Genes Dev 2000; 14:2938-2943.

48 Mähönen AP, Bishopp A, Higuchi M, et al. Cytokinin signaling and its inhibitor AHP6 regulate cell fate during vascular development. Science 2006; 311:94-98.

49 Kakimoto T. CKI1, a histidine kinase homolog implicated in cytokinin signal transduction. Science 1996; 274:982-985.

50 Kim HJ, Ryu H, Hong SH, et al. Cytokinin-mediated control of leaf longevity by AHK3 through phosphorylation of ARR2 in Arabidopsis. Proc Natl Acad Sci USA 2006; 103:814-819.

51 Hutchison CE, Kieber JJ. Cytokinin signaling in Arabidopsis.
Plant Cell 2002; 14:S47-S59.

52 Reed JW. Roles and activities of AUX/IAA proteins in Arabidopsis. Trends Plant Sci 2001; 6:420-425.

53 Ferreira FJ, Kieber JJ. Cytokinin signaling. Curr Opin Plant Biol 2005; 8:518-525.

54 Smalle J, Kurepa J, Yang P, et al. Cytokinin growth responses in Arabidopsis involve the 26S proteasome subunit RPN12. Plant Cell 2002; 14:17-32.

55 Vogel JP, Schuerman P, Woeste K, Brandstatter I, Kieber JJ. Isolation and characterization of Arabidopsis mutants defective in the induction of ethylene biosynthesis by cytokinin. Genetics 1998; 149:417-427.

56 Lee DJ, Kim S, Ha YM, Kim J. Phosphorylation of Arabidopsis response regulator 7 (ARR7) at the putative phosphoaccepting site is required for ARR7 to act as a negative regulator of cytokinin signaling. Planta 2008; 227:577-587.

57 Murashige T, Skoog F. A revised medium for rapid growth and bioassays with tobacco tissue culture. Physiol Plant 1962; 15:473-497.

58 Clough SJ, Bent AF. Floral dip: a simplified method for Agrobacterium-mediated transformation of Arabidopsis thaliana. Plant J 1998; 16:735-743.

59 Sun J, Niu QW, Tarkowski P, et al. The Arabidopsis AtIPT8/ $P G A 22$ gene encodes an isopentenyl transferase that is involved in de novo cytokinin biosynthesis. Plant Physiol 2003; 131:167-176.

60 Feng H, Chen Q, Feng J, et al. Functional characterization of the Arabidopsis eukaryotic translation initiation factor 5A-2 that plays a crucial role in plant growth and development by regulating cell division, cell growth, and cell death. Plant Physiol 2007; 144:1531-1545.

(Supplementary information is linked to the online version of the paper on the Cell Research website.) 